\title{
SYMMETRY-PRESERVING REVERSIBLE INTEGER-TO-INTEGER WAVELET TRANSFORMS
}

\author{
Michael D. Adams and Rabab Ward \\ Dept. of Elec. and Comp. Eng., University of British Columbia, 2356 Main Mall, Vancouver, BC, Canada V6T 1Z4 \\ mdadams@ieee.org and rababw@ece.ubc.ca
}

\begin{abstract}
Studied are two lifting-based families of symmetry-preserving reversible integer-to-integer wavelet transforms. The transforms from both of these families are shown to be compatible with symmetric extension, which permits the treatment of arbitrary length signals in a nonexpansive manner. Throughout this work, particularly close attention is paid to rounding functions, and the properties that they must possess in various instances. Symmetric extension is also shown to be equivalent to constant per-lifting-step extension in certain circumstances.
\end{abstract}

\section{INTRODUCTION}

Lifting-based reversible integer-to-integer wavelet transforms [1, 2] have become a popular tool in signal coding applications. In such applications, however, it is often desirable to employ transforms that preserve symmetry. For example, symmetry-preserving transforms have the advantage of being compatible with symmetric extension techniques, allowing signals of arbitrary length to be handled in a nonexpansive manner [3]. (As a matter of terminology, a transform is said to be nonexpansive if its application to a signal of length $N$ always yields a result that can be completely characterized by no more than $N$ transform coefficients.) Fortunately, using the lifting scheme, one can construct transforms that are not only reversible and integer to integer, but also symmetry preserving.

In this paper, we study two families of symmetry-preserving reversible integer-to-integer wavelet transforms. Both of these families of transforms are shown to be compatible with symmetric extension, facilitating the treatment of arbitrary length signals in a nonexpansive manner. In our treatment, we pay particularly close attention to the rounding functions involved, emphasizing the properties that such functions must possess in various circumstances. Lastly, we examine the relationship between symmetric extension and per-lifting-step extension.

\section{NOTATION AND OTHER PRELIMINARIES}

Before proceeding further, a short digression concerning the notation used in this paper is appropriate. The symbols $\mathbb{Z}$ and $\mathbb{R}$ denote the sets of integer and real numbers, respectively. For $\alpha \in \mathbb{R}$, the notation $\lfloor\alpha\rfloor$ denotes the largest integer not more than $\alpha$ (i.e., the floor function), and the notation $\lceil\alpha\rceil$ denotes the smallest integer not less than $\alpha$ (i.e., the ceiling function). One can show that the floor and ceiling functions are related as follows:

$$
\lceil\alpha\rceil=-\lfloor-\alpha\rfloor \quad \text { for all } \alpha \in \mathbb{R} \text {. }
$$

This work was supported by the Natural Sciences and Engineering Research Council of Canada.
The signum, truncation, rounding-away-from-zero (RAFZ), and mod functions are defined, respectively, as

$$
\begin{gathered}
\operatorname{sgn} \alpha \triangleq\left\{\begin{array} { l l } 
{ 1 } & { \text { for } \alpha > 0 } \\
{ 0 } & { \text { for } \alpha = 0 , } \\
{ - 1 } & { \text { for } \alpha < 0 }
\end{array} \quad \text { trunc } \alpha \triangleq \left\{\begin{array}{ll}
\lfloor\alpha\rfloor & \text { for } \alpha \geq 0 \\
\lceil\alpha\rceil & \text { for } \alpha<0
\end{array},\right.\right. \\
\operatorname{rafz} \alpha \triangleq\left\{\begin{array}{ll}
\lceil\alpha\rceil & \text { for } \alpha \geq 0 \\
\lfloor\alpha\rfloor & \text { for } \alpha<0
\end{array},\right. \text { and } \\
\bmod (x, y) \triangleq x-y\lfloor x / y\rfloor, \quad \text { where } \alpha \in \mathbb{R} \text { and } x, y \in \mathbb{Z} . \\
\text { A rounding operator } \Omega \text { is said to be integer-shift invariant if } \\
\quad \Omega(\alpha+x)=\Omega(\alpha)+x \text { for all } \alpha \in \mathbb{R} \text { and all } x \in \mathbb{Z} . \\
\text { Similarly, a rounding operator } \Omega \text { is said to be odd if } \\
\Omega(\alpha)=-\Omega(-\alpha) \text { for all } \alpha \in \mathbb{R} .
\end{gathered}
$$

One can show that a rounding operator cannot be both odd and integer-shift invariant [4]. In passing, we note that the floor and ceiling functions are integer-shift invariant (but not odd), while the trunc and rafz functions are odd (but not integer-shift invariant). All rounding operators considered in this paper are tacitly assumed to be memoryless, time invariant, and leave integer values unchanged. Any (reasonable) rounding operator will preserve signal symmetry (but not necessarily signal antisymmetry), while any odd rounding operator will preserve both symmetry and antisymmetry.

\section{TRANSFORM FAMILIES}

In this paper, we consider two families of symmetry-preserving reversible integer-to-integer wavelet transforms. Both are derived from the lifting-based parameterizations of linear-phase filter banks presented in [5], and have the general form shown in Fig. 1. In the figure, the $\left\{\Omega_{k}\right\}$ and $\left\{\mho_{k}\right\}$ are rounding operators. As we will demonstrate, by choosing the lifting filters $\left\{A_{k}\right\}$ and $\left\{B_{k}\right\}$ wisely, one can construct filter banks that not only yield symmetric/antisymmetric subband signals (i.e., $y_{0}[n]$ and $y_{1}[n]$ ) for an appropriately chosen input signal (i.e., $x[n]$ ), but also yield intermediate signals (i.e., $\left\{u_{k}[n]\right\},\left\{v_{k}[n]\right\}$ ) that are all or mostly symmetric/antisymmetric.

The first family of reversible integer-to-integer wavelet transforms is associated with a linear-phase filter bank having oddlength analysis/synthesis filters, and has been described in [5]. For convenience, we will refer to this as the odd-length analysis/synthesis filter (OLASF) parameterization. In this case, the lifting step filters are chosen to have transfer functions of the form:

$$
\begin{aligned}
& A_{k}(z)=\sum_{i=0}^{\left(K_{k}-2\right) / 2} a_{k, i}\left(z^{-i}+z^{i+1}\right) \quad \text { and } \\
& B_{k}(z)=\sum_{i=0}^{\left(L_{k}-2\right) / 2} b_{k, i}\left(z^{-i-1}+z^{i}\right)
\end{aligned}
$$

for $k=0,1, \ldots, \lambda-1$, where the $\left\{K_{k}\right\}$ and $\left\{L_{k}\right\}$ are all even integers. The $\left\{\Omega_{k}\right\}_{k=0}^{\lambda-1}$ and $\left\{\mho_{k}\right\}_{k=0}^{\lambda-1}$ are chosen to be any arbitrary rounding operators.

The second family of reversible integer-to-integer wavelet transforms is associated with a linear-phase filter bank having even- 


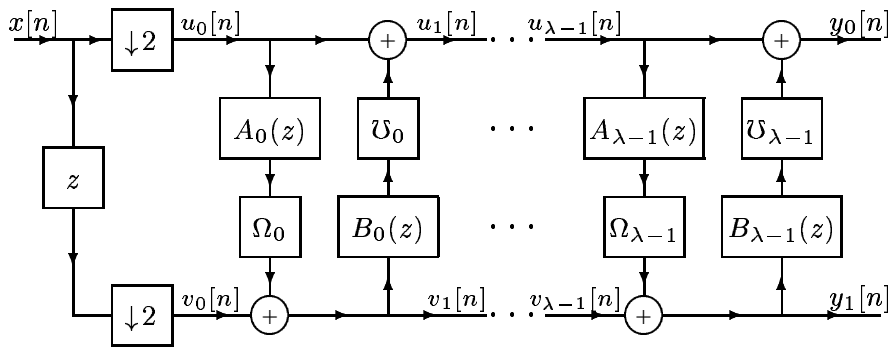

(a)

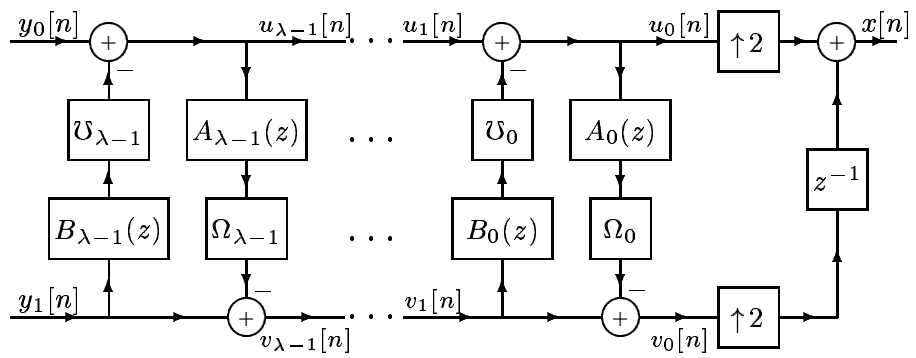

(b)

Fig. 1. Lifting-based realization of reversible integer-to-integer wavelet transform. (a) Analysis filter bank and (b) synthesis filter bank.

length analysis/synthesis filters, and has been described in [5]. For convenience, we will refer to this as the even-length analysis/synthesis filter (ELASF) parameterization. In this case, the lifting step filters are chosen to have transfer functions of the form:

$$
\begin{gathered}
A_{0}(z)=-1, \quad B_{0}(z)=\frac{1}{2}+\hat{B}_{0}(z), \\
\hat{B}_{0}(z)=\sum_{i=1}^{\left(L_{0}-1\right) / 2} \hat{b}_{0, i}\left(z^{-i}-z^{i}\right), \\
A_{k}(z)=\sum_{i=1}^{\left(K_{k}-1\right) / 2} a_{k, i}\left(z^{-i}-z^{i}\right) \quad \text { for } k=1,2, \ldots, \lambda-1, \\
B_{k}(z)=\sum_{i=1}^{\left(L_{k}-1\right) / 2} b_{k, i}\left(z^{-i}-z^{i}\right) \quad \text { for } k=1,2, \ldots, \lambda-1,
\end{gathered}
$$

where the $\left\{K_{k}\right\}$ and $\left\{L_{k}\right\}$ are all odd integers. The operator $\Omega_{0}$ is simply the identity (since the output of the filter $A_{0}$ is always an integer), and the rounding operator $\mho_{0}$ is chosen to be integer-shift invariant. The rounding operators $\left\{\Omega_{k}\right\}_{k=1}^{\lambda-1}$ are chosen to be odd, and the $\left\{\mho_{k}\right\}_{k=1}^{\lambda-1}$ are chosen arbitrarily.

\section{TRANSFORMS AND SYMMETRIC EXTENSION}

In the previous section, we introduced the OLASF and ELASF families of reversible integer-to-integer wavelet transforms. Now, we show that the transforms from these two families can be used with symmetric extension in order to handle signals of arbitrary length in a nonexpansive manner.

Consider a filter bank of the form shown in Fig. 1 that is constrained to be of the OLASF type. Suppose that we are given a signal $\hat{x}[n]$ defined for $n=0,1, \ldots, N-1$. We then choose $x[n]$, the input to the analysis filter bank, as the following symmetric extension of $\hat{x}[n]$ :

$x[n]=\hat{x}[\min (\bmod (n, 2 N-2), 2 N-2-\bmod (n, 2 N-2))]$

(i.e., $x[n]$ is defined such that $x[n]=x[n+2 N-2]$ and $x[n]=$ $x[-n])$. Evidently, $x[n]$ is $(2 N-2)$-periodic and symmetric about 0 and $N-1$ (with additional pairs of symmetry points following from periodicity). From the properties of downsampling, one can show that $u_{0}[n]$ is $(N-1)$-periodic and symmetric about 0 and
$\frac{N-1}{2}$, and $v_{0}[n]$ is $(N-1)$-periodic and symmetric about $-\frac{1}{2}$ and $\frac{N-2}{2}$. Consider now the first lifting step (associated with filter $A_{0}$ ). The filter $A_{0}$ has a group delay of $-\frac{1}{2}$ and the rounding operator $\Omega_{0}$ preserves symmetry, so the adder is summing two $(N-1)$ periodic symmetric signals with the same symmetry centers. Thus, the adder output, $v_{1}[n]$, is also $(N-1)$-periodic and symmetric with the same symmetry centers, namely $-\frac{1}{2}$ and $\frac{N-2}{2}$. Consider now the second lifting step (associated with filter $B_{0}^{2}$ ). The filter $B_{0}$ has a group delay of $\frac{1}{2}$ and the rounding operator $\mho_{0}$ preserves symmetry, so both adder inputs are $(N-1)$-periodic symmetric signals with the same symmetry centers. Therefore, the adder output, $u_{1}[n]$, must also be $(N-1)$-periodic and symmetric with the same symmetry centers, namely 0 and $\frac{N-1}{2}$. By repeating the above reasoning for each of the remaining lifting steps, we have that the $\left\{u_{k}[n]\right\}_{k=0}^{\lambda-1}$ and $y_{0}[n]$ are all $(N-1)$-periodic and symmetric about 0 and $\frac{N-1}{2}$. Likewise, the $\left\{v_{k}[n]\right\}_{k=0}^{\lambda-1}$ and $y_{1}[n]$ are all $(N-1)$-periodic and symmetric about $-\frac{1}{2}$ and $\frac{N-2}{2}$.

By examining the form of the various signals we note that the $\left\{u_{k}[n]\right\}_{k=0}^{\lambda-1}$ and $y_{0}[n]$ are completely characterized by their samples at indices $0,1, \ldots,\left\lfloor\frac{N-1}{2}\right\rfloor$, and the $\left\{v_{k}[n]\right\}_{k=0}^{\lambda-1}$ and $y_{1}[n]$ are completely characterized by their samples at indices $0,1, \ldots$, $\left\lfloor\frac{N-2}{2}\right\rfloor$. Consequently, for even $N$, we require $\frac{N}{2}$ samples to represent each of $y_{0}[n]$ and $y_{1}[n]$, and for odd $N$, we need $\frac{N+1}{2}$ samples to represent $y_{0}[n]$ and $\frac{N-1}{2}$ samples to represent $y_{1}[n]$. In either case, a total of $N$ samples is required, and the resulting transform is, therefore, nonexpansive.

Consider a filter bank of the form shown in Fig. 1 that is constrained to be of the ELASF type. Suppose that we are given a signal $\hat{x}[n]$ defined for $n=0,1, \ldots, N-1$. We then choose $x[n]$, the input to the analysis filter bank, as the following symmetric extension of $\hat{x}[n]$ :

$$
x[n]=\hat{x}[\min (\bmod (n, 2 N), 2 N-1-\bmod (n, 2 N))]
$$

(i.e., $x[n]$ is defined such that $x[n]=x[n+2 N]$ and $x[n]=$ $x[-1-n]$ ). For the time being, we assume that $u_{1}[n]$ is $N$-periodic and symmetric about $-\frac{1}{2}$ and $\frac{N-1}{2}$, and $v_{1}[n]$ is $N$-periodic and antisymmetric with the same symmetry points as $u_{1}[n]$. These assumptions will be proven valid in Section 5. Consider now the third lifting step (associated with filter $A_{1}$ ). The filter $A_{1}$ has a group delay of 0 and the rounding operator $\Omega_{1}$ preserves antisymmetry (since it is odd, by assumption), so the adder is summing two $N$-periodic antisymmetric signals with the same symmetry centers. Thus, the adder output is also $N$-periodic and antisymmetric with the same symmetry centers. Consider the fourth lifting step (associated with filter $B_{1}$ ). The filter $B_{1}$ has a group delay of 0 and the rounding operator $\mho_{1}$ preserves symmetry, so the adder is summing two $N$-periodic symmetric signals with the same symmetry centers. Thus, the adder output is also $N$-periodic and symmetric with the same symmetry centers. It is important to note that $\mho_{1}$ need not be odd, contrary to the suggestions of some, in the case of similar parameterizations (e.g., [6]). By repeating the above reasoning for each of the remaining lifting steps, we have that the $\left\{u_{k}[n]\right\}_{k=1}^{\lambda-1}$ and $y_{0}[n]$ are $N$-periodic and symmetric with symmetry centers $-\frac{1}{2}$ and $\frac{N-1}{2}$, and the $\left\{v_{k}[n]\right\}_{i=1}^{\lambda-1}$ and $y_{1}[n]$ are $N$-periodic and antisymmetric with the same symmetry centers.

By examining the form of the various signals, we note that the $\left\{u_{k}[n]\right\}_{k=1}^{\lambda-1}$ and $y_{0}[n]$ are completely characterized by their samples at indices $0,1, \ldots,\left\lfloor\frac{N-1}{2}\right\rfloor$ and the $\left\{v_{k}[n]\right\}_{k=1}^{\lambda-1}$ and $y_{1}[n]$ are completely characterized by their samples at indices $0,1, \ldots$, $\left\lfloor\frac{N-2}{2}\right\rfloor$. Consequently, for even $N$, we require $\frac{N}{2}$ samples to represent each of $y_{0}[n]$ and $y_{1}[n]$, and for odd $N$, we require $\frac{N+1}{2}$ samples to represent $y_{0}[n]$ and $\frac{N-1}{2}$ samples to represent $y_{1}[n]$. In either case, regardless of the parity of $N$, a total of $N$ samples is required, and the resulting transform is, therefore, nonexpansive. 


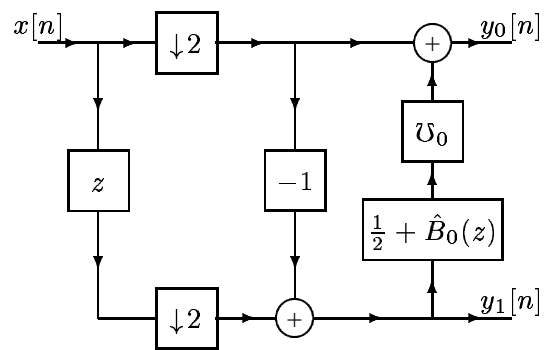

Fig. 2. Base analysis filter bank for the ELASF family of transforms.

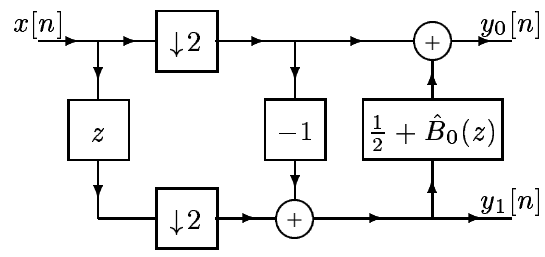

Fig. 3. Linear version of the base analysis filter bank for the ELASF family of transforms.

\section{ELASF BASE FILTER BANK}

Consider a filter bank of the form shown in Fig. 1 that is constrained to be of the ELASF type with $\lambda=1$. The analysis side of such a filter bank has the form illustrated in Fig. 2. In the previous section, we made certain assumptions concerning the form of $y_{0}[n]$ and $y_{1}[n]$ supposing that $x[n]$ is generated using (3). We will now prove our earlier assumptions to be valid.

We begin by considering a slightly different filter bank of the form shown in Fig. 3. For this filter bank, let us denote the transfer functions of the lowpass and highpass analysis filters as $H_{0}(z)$ and $H_{1}(z)$, respectively. Using the noble identities, we can show that

$$
H_{0}(z)=\frac{1}{2}(z+1)+(z-1) \hat{B}_{0}\left(z^{2}\right) \quad \text { and } \quad H_{1}(z)=z-1 \text {. }
$$

Due to the form of $\hat{B}_{0}(z)$, it follows that the filter $H_{0}$ has an impulse response symmetric about $-\frac{1}{2}$. Clearly, the filter $H_{1}$ has an impulse response that is antisymmetric about $-\frac{1}{2}$. Consequently, if $x[n]$ is generated using (3), we have that $y_{0}[n]$ is $N$-periodic and symmetric about $-\frac{1}{2}$ and $y_{1}[n]$ is $N$-periodic and antisymmetric about $-\frac{1}{2}$.

Now, suppose that we round the lowpass subband output, so as to obtain the system shown in Fig. 4. For any (non-pathological) rounding function, this process will maintain signal symmetry. Moreover, if the rounding operator, $\mho_{0}$, is integer-shift invariant, we can equivalently move the rounding operator to the input side of the adder, resulting in the system in Fig. 2. Consequently, in the case of the system in Fig. 2, if the input $x[n]$ has the form of (3), $y_{0}[n]$ must be $N$-periodic and symmetric about $-\frac{1}{2}$ and $y_{1}[n]$ must be $N$-periodic and antisymmetric about $-\frac{1}{2}$. This is the result that we initially sought to prove.

As demonstrated above, the system of Fig. 4 has the symmetrypreserving properties that we desire, regardless of whether the rounding operator $\mho_{0}$ is integer-shift invariant. If $\mho_{0}$ is not integershift invariant, however, it is not clear that the resulting transform necessarily has an inverse. In fact, one can easily prove that some such choices of $\mho_{0}$ do not lead to reversible transforms (e.g., $\mho_{0}(x)=\operatorname{trunc} x$ ). This said, however, at least one common rounding operator does yield a reversible transform. In particular, if $\mho_{0}$ is chosen as $\mho_{0}(x)=\operatorname{rafz} x$, the resulting transform is reversible, an assertion that we will now prove.

Consider a network of the form shown in Fig. 5, where the

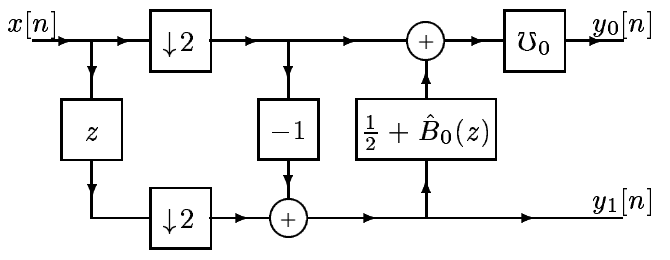

Fig. 4. Modified base analysis filter bank for the ELASF family of transforms.

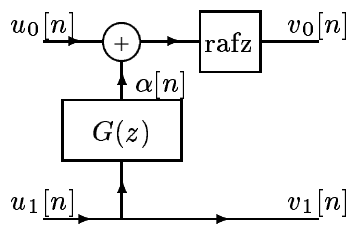

Fig. 5. Network consisting of a ladder step and rounding function.

$\left\{u_{k}[n]\right\}$ and $\left\{v_{k}[n]\right\}$ are integer-valued sequences. Clearly, if this network can be inverted, so can the network in Fig. 4. For convenience, we define the quantities $u \triangleq u_{0}[n], v \triangleq v_{0}[n], \alpha \triangleq \alpha[n]$. The network in Fig. 5 can be inverted if we can solve for $u$ in terms of $v$ and $\alpha$ in the equation

$$
v=\operatorname{rafz}(u+\alpha)
$$

We now proceed to manipulate the preceding equation as follows:

$$
\begin{aligned}
v & =\operatorname{rafz}(u+\alpha) \\
& = \begin{cases}\lceil u+\alpha\rceil & \text { for } u+\alpha \geq 0 \\
\lfloor u+\alpha\rfloor & \text { for } u+\alpha<0\end{cases} \\
& = \begin{cases}u+\lceil\alpha\rceil & \text { for } u+\alpha \geq 0 \\
u+\lfloor\alpha\rfloor & \text { for } u+\alpha<0\end{cases}
\end{aligned}
$$

Solving for $u$ in the above equation, we obtain

$$
u= \begin{cases}v-\lceil\alpha\rceil & \text { for } u+\alpha \geq 0 \\ v-\lfloor\alpha\rfloor & \text { for } u+\alpha<0\end{cases}
$$

From the definition of the rafz function, however, we know that $\operatorname{sgn}(\operatorname{rafz} \alpha)=\operatorname{sgn} \alpha$ for all $\alpha \in \mathbb{R}$ (i.e., the rafz function preserves signedness). Consequently, we have that $\operatorname{sgn}(u+\alpha)=$ $\operatorname{sgn} v$. Using this fact and algebraic manipulation, we can rewrite (4) as follows:

$$
\begin{aligned}
u & = \begin{cases}v-\lceil\alpha\rceil & \text { for } v \geq 0 \\
v-\lfloor\alpha\rfloor & \text { for } v<0\end{cases} \\
& = \begin{cases}\lfloor v-\alpha\rfloor & \text { for } v \geq 0 \\
\lceil v-\alpha\rceil & \text { for } v<0 .\end{cases}
\end{aligned}
$$

Thus, we have solved for $u$ in terms of $v$ and $\alpha$. Consequently, we can invert the network in Fig. 5, and, in turn, the network in Fig. 4 as well.

\section{RELATIONSHIP BETWEEN SYMMETRIC EXTENSION AND PER-LIFTING-STEP EXTENSION}

Although symmetric extension is very popular, others extension schemes also exist. For example, one common extension technique used in conjunction with lifting-based transforms is the perlifting-step extension method. This technique is mentioned briefly in [7] and used in the SBTLIB [8] and JasPer [9] software. More recently, this method has also been described in $[6,10]$ (under the name of "iterated extension").

With per-lifting-step extension, signal extension is performed at the input to each lifting step filter rather than being performed 


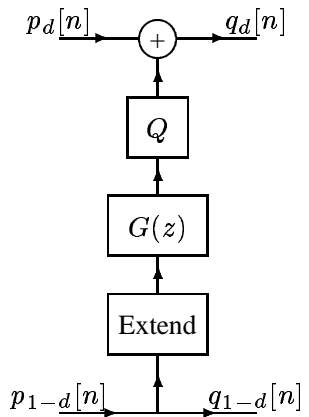

(a)

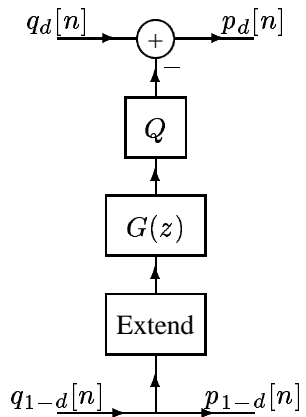

(b)
Fig. 6. Structure of a lifting step in the case of per-lifting-step extension. (a) Forward lifting step and (b) inverse lifting step.

at the input to the filter bank. That is, each lifting step on the analysis side of the filter bank has the form shown in Fig. 6(a). In the diagram, $p_{0}[n]$ and $q_{0}[n]$ represent intermediate lowpass channel signals, $p_{1}[n]$ and $q_{1}[n]$ represent intermediate highpass channel signals, and $Q$ is a rounding operator. The parameter $d$ determines the channel modified by the lifting step. On the synthesis side of the filter bank, the lifting step of Fig. 6(a) has a corresponding inverse of the form shown in Fig. 6(b). Clearly, the same extension values can be generated on both the analysis and synthesis sides (since $p_{1-d}[n]=q_{1-d}[n]$ ). Thus, this scheme can generate reversible transforms.

Consider again a system of the form shown in Fig. 1. In Section 4, we carefully examined the symmetry properties of the signals $\left\{u_{k}[n]\right\}_{k=0}^{\lambda-1},\left\{v_{k}[n]\right\}_{k=0}^{\lambda-1}, y_{0}[n]$, and $y_{1}[n]$. In the OLASF case, all of these signals are symmetric, while in the ELASF case, all of these signals are symmetric/antisymmetric, except $u_{0}[n]$ and $v_{0}[n]$. Therefore, we can equivalently define symmetric extension in terms of per-lifting-step extension in which case the input to each lifting step filter is extended by symmetric extension (with the appropriate choice of symmetry type and centers).

Suppose that we have a filter bank of the form shown in Fig. 1 that is constrained to be of the OLASF type with $K_{k} \leq 2$ and $L_{k} \leq 2$ for $k=0,1, \ldots, \lambda-1$. In this case, we assert that symmetric extension is equivalent to constant per-lifting-step extension (where the lifting step filter input is extended to the left by repeating its leftmost sample and to the right by repeating its rightmost sample). In the case of both symmetric extension and constant per-lifting-step extension, the signals $\left\{u_{k}[n]\right\}_{k=0}^{\lambda-1}$ and $y_{0}[n]$ are completely characterized by their samples at indices $n=0,1, \ldots$, $\left\lfloor\frac{N-1}{2}\right\rfloor$ and the signals $\left\{v_{k}[n]\right\}_{k=0}^{\lambda-1}$ and $y_{1}[n]$ are completely characterized by their samples at indices $n=0,1, \ldots,\left\lfloor\frac{N-2}{2}\right\rfloor$. Furthermore, both extension methods yield the same $u_{0}[n]$ and $v_{0}[n]$ for $n$ over their characteristic sample indices.

Consider the lifting steps involving the filters $\left\{B_{k}\right\}$. When filtering with the filter $B_{k}, v_{k+1}[n]$ always requires left extension by one sample in order to obtain the value for $v_{k+1}[-1]$. In the case of symmetric extension, since a symmetry center of $v_{k+1}[n]$ is $-\frac{1}{2}$, the sample obtained by extension is equal to $v_{k+1}[0]$. Clearly, this is the same result obtained by constant per-lifting-step extension. If $N$ is odd, $v_{k+1}[n]$ must also be right extended by one sample in order to obtain the value for $v_{k+1}\left[\frac{N-1}{2}\right]$ (since one fewer sample is associated with $v_{k+1}[n]$ than $u_{k+1}[n]$ ). In the case of symmetric extension, the symmetry center $\frac{N-2}{2}$ is an odd multiple of $\frac{1}{2}$, so the sample obtained by extension is equal to $v_{k+1}\left[\frac{N-3}{2}\right]$. Again, this is the same result that is obtained from constant per-liftingstep extension. Thus, symmetric extension is equivalent to constant per-lifting-step extension for the lifting steps involving the filters $\left\{B_{k}\right\}$.
Consider the lifting steps involving the filters $\left\{A_{k}\right\}$. When filtering with the filter $A_{k}, u_{k}[n]$ only ever requires right extension by one sample (if at all) in order to obtain the value for $u_{k}\left[\left\lfloor\frac{N+1}{2}\right\rfloor\right]$. Suppose first that $N$ is even. In the case of symmetric extension, the symmetry center of $u_{k}[n]$ at $\frac{N-1}{2}$ is an odd multiple of $\frac{1}{2}$, so the sample obtained by extension is equal to $u_{k}\left[\frac{N-2}{2}\right]$. This, however, is the same result obtained by constant per-lifting-step extension. Finally, if $N$ is odd, one less sample needs to be computed for $v_{k}[n]$ than $u_{k}[n]$, and $u_{k}[n]$ need not be extended at all. Thus, symmetric extension is equivalent to constant per-lifting-step extension for the lifting steps involving the filters $\left\{A_{k}\right\}$.

Combining the above results for both sets of lifting filters, we see that constant per-lifting-step extension is equivalent to symmetric extension for the specific case considered. Since constant per-lifting-step extension is typically easier to implement than symmetric extension, this equivalence is potentially quite useful. For example, both of the filter banks defined in the JPEG-2000 Part-1 standard (i.e., ISO/IEC 15444-1:2001 [2]) are of the form assumed above. Therefore, one can exploit the equivalence between symmetric extension and constant per-lifting-step extension in order to simplify JPEG-2000 codec implementations. For example, this equivalence has been employed by the JasPer software [9] since at least version 0.044 .

\section{CONCLUSIONS}

Two families of symmetry-preserving reversible integer-to-integer wavelet transforms were studied (i.e., the OLASF and ELASF families). We showed that the transforms from both families are compatible with symmetric extension, and can be used to handle signals of arbitrary length in a nonexpansive manner. For OLASF systems with length-2 lifting filters, we showed that symmetric extension is equivalent to constant per-lifting-step extension. This fact can be exploited in order to reduce the complexity of JPEG2000 Part-1 codec implementations.

\section{REFERENCES}

[1] A. R. Calderbank, I. Daubechies, W. Sweldens, and B.-L. Yeo, "Wavelet transforms that map integers to integers," Applied and Computational Harmonic Analysis, vol. 5, no. 3, pp. 332369, July 1998.

[2] ISO/IEC, ISO/IEC 15444-1: Information technology-JPEG 2000 image coding system-Part 1: Core coding system, 2001.

[3] C. M. Brislawn, "Preservation of subband symmetry in multirate signal coding," IEEE Trans. on Signal Processing, vol. 43, no. 12, pp. 3046-3050, Dec. 1995.

[4] M. D. Adams and F. Kossentini, "Generalized S transform," in Proc. of IEEE ICASSP, 2001, pp. 1749-1752.

[5] M. D. Adams and F. Kossentini, "Low-complexity reversible integer-to-integer wavelet transforms for image coding," in Proc. of IEEE Pacific Rim Conference, Victoria, BC, Canada, Aug. 1999, pp. 177-180.

[6] C. Brislawn and B. Wohlberg, "Boundary extensions and reversible implementation for half-sample symmetric fi lter banks," ISO/IEC JTC 1/SC 29/WG 1 N 2119, Mar. 2001.

[7] M. D. Adams and F. Kossentini, "Reversible integer-to-integer wavelet transforms for image compression: Performance evaluation and analysis," IEEE Trans. on Image Processing, vol. 9, no. 6, pp. 1010-1024, June 2000.

[8] M. D. Adams and F. Kossentini, "SBTLIB: A fexible computation engine for subband transforms," ISO/IEC JTC 1/SC 29/WG 1 N 867, June 1998.

[9] M. D. Adams and F. Kossentini, "JasPer: A software-based JPEG-2000 codec implementation," in Proc. of IEEE ICIP, Vancouver, BC, Canada, Oct. 2000, vol. 2, pp. 53-56.

[10] C. Brislawn, S. Mniszewski, M. Pal, A. Percus, B. Wohlberg, T. Acharya, P.-S. Tsai, and M. Lepley, "Even-length fi lter bank option,” ISO/IEC JTC 1/SC 29/WG 1 N 2209, July 2001. 